\title{
Análise da Publicação Científica de enfermeiras assistenciais com apoio de um núcleo de pesquisa em enfermagem
}

\section{Analysis of the Scientific Production of clinical nurses supported by a nursing research advisory body}

\author{
Maria Helena Dantas de Menezes Guariente ${ }^{1}$; Márcia Maria Fontão Zago²; Zeneide Soubhia ${ }^{3}$; Neusi \\ Aparecida Navas Berbel ${ }^{4}$; Maria do Carmo Lourenço Haddad ${ }^{5}$
}

\section{Resumo}

É cada vez mais ressaltada a importância da produção científica, associada à crença de que, quando divulgada, informa e possibilita aprendizagem e inovações na assistência de enfermagem. O artigo apresenta a análise da produção científica das enfermeiras assistenciais do Hospital Universitário Regional do Norte do Paraná, a partir da implantação do Núcleo de Pesquisa em Enfermagem (NUPE), referente ao período de 1999 a 2002. Os dados foram coletados em fontes documentais primárias do NUPE. Verificouse que os enfermeiros elaboraram e encaminharam para publicação 63 trabalhos científicos, sendo 36 publicados. A publicação efetivada perfaz a média de 1,56 artigos por enfermeira e 0,39 publicações por enfermeira/ano. Os artigos, em grande parte, construídos coletivamente e publicados em diversos veículos de divulgação, relacionam-se ao Campo Temático Assistencial e Organizacional. Seis trabalhos foram premiados em eventos científicos. A pesquisa, quando apoiada pela organização e desejada pela enfermeira, consubstancia-se como estratégia científico-pedagógica na formação permanente de enfermeiras assistenciais e agrega repercussões positivas para a assistência prestada.

Palavras-chave: Pesquisa em enfermagem. Enfermeiras clínicas. Publicações periódicas.

\footnotetext{
Abstract

The importance of scientific production is more and more emphasized, together with the belief that, when it is disseminated, it informs and enables learning and innovations in nursing assistance. The article presents the analysis of the scientific production of the Clinical Nurses of the Regional University Hospital of Northern Paraná, based on activities carried out at the Nursing Research Nucleus (NUPE),

${ }^{1}$ Professora Adjunta do Departamento de Enfermagem da Universidade Estadual de Londrina, Doutora em Enfermagem. E-mail: mhguariente@onda.com.br

${ }^{2}$ Professora Titular da Escola de Enfermagem de Ribeirão Preto - USP, Doutora em Enfermagem.

${ }^{3}$ Professora Adjunta do Departamento de Enfermagem da Universidade Estadual de Londrina, Doutora em Enfermagem.

${ }^{4}$ Professora Adjunta do Departamento de Educação da Universidade Estadual de Londrina, Doutora em Educação.

${ }^{5}$ Professora Adjunta do Departamento de Enfermagem da Universidade Estadual de Londrina, Doutora em Enfermagem.
} 
from 1999 to 2002. The data were gathered from NUPE's primary documentary sources. It was verified that the nurses produced and submitted 63 scientific articles for publication, having 36 been published, which accounts for the average of 1.56 article per nurse and 0.39 publications per nurse/year. Most of the articles were collectively produced and published in different types of publications. They are related to the Care and Organizational Thematic Field. Six articles were awarded a prize in scientific events. The research, when supported by the organization and wished by the nurse, becomes a scientific-pedagogical strategy used in the continuing education of clinical nurses and aggregates positive repercussions to the assistance given.

Key words: Nursing research. Clinical nurses. Periodicals

\section{Introdução}

A literatura acerca da temática pesquisa entre enfermeiras assistenciais mostra o grande embate enfrentado por estes profissionais entre o querer e o poder efetivá-la, tanto no plano pessoal como institucional. No entanto, todos sentem a necessidade da realização dessa prática, por ser ela necessária ao desenvolvimento do profissional e da profissão (CASTILHO; KURCGANT, 1999; CASSIANI; PASSARELLI, 1999; CALIRI, 2002).

Alguns autores referem que existem ainda muitos limites para a realização de pesquisas, os quais provêm da deficitária formação universitária, do difícil acesso à pós-graduação, da falta do apoio das instituições empregadoras e do pouco interesse e iniciativa dos profissionais (LOPES, 1993; HADDAD; GUARIENTE, 2000; CALIRI, 2002; DYNIEWICZ, 2003).

Costa e Carvalho (2001) relatam que as enfermeiras assistenciais devem ser mais estimuladas pelas instituições para que relatem suas experiências, efetuem pesquisas e publiquem o resultado das mesmas. Além disso, as instituições devem valorizar, nos planos de carreira, estas atividades profissionais, assim como criar condições para que estes profissionais dêem sua contribuição à comunidade científica.

Acontecimentos recentes são as parcerias entre instituições de ensino e empregadoras da saúde, visando ao aprimoramento do seu pessoal, apoio às enfermeiras na efetivação de pesquisas, com a formação de grupos de estudo e núcleos de pesquisa entre outras atividades. Vários grupos, comissões e núcleos de pesquisa vêm sendo criados com a finalidade de estimular as enfermeiras e auxiliá-las no desenvolvimento e na divulgação de pesquisas, como importante recurso no seu processo de trabalho (TASCA et al., 1996).

Além disso, nos dias atuais, vivemos sob a influência da expressão: Publicar ou morrer! Pesquisar ou padecer! Essa expressão, em evidência entre as enfermeiras norte-americanas e ouvida por nós no ano de 2000, no Seminário Internacional sobre Publicação em Periódicos de Enfermagem, vem-se tornando um desafio profissional também entre as enfermeiras do nosso País.

A pesquisa começa a tornar-se uma práxis possível para essas profissionais, situação já há algum tempo anunciada como primordial e inspirada na intenção de desmistificar a sua produção e consumo, daí resultando a ampliação dos conhecimentos da ciência do cuidado (CIANCIARULLO, 1992).

Seguindo essas premissas, a Diretoria de Enfermagem(DE) do Hospital Universitário Regional do Norte do Paraná (HURNP) criou, em 1999, o Núcleo de Pesquisa de Enfermagem (NUPE). Esse núcleo constituiu-se como um serviço de Assessoria da Diretoria de Enfermagem, tendo como coordenadora uma docente do Departamento de Enfermagem do Centro de Ciências da Saúde da Universidade Estadual de Londrina(UEL).

ONUPE foi implantadocom o objetivo de oferecer apoio científico e administrativo para a execução, apresentação e publicação dos trabalhos científicos. 
Após quatro anos de funcionamento deste serviço, período marcado por muitas atividades, desafios e conquistas, o Núcleo, como serviço inovador na área e como estratégia organizacional, incentivou a produção científica dos enfermeiros assistenciais?

A pesquisa é sentida como possível e necessária para subsidiar o desenvolvimento das competências quantitativa e qualitativa das enfermeiras, na busca de soluções para os problemas do cuidar. Agregada a esse contexto, ela traz a expectativa de produzir impacto e mudanças na qualidade da assistência nos serviços prestados à população.

A pesquisa é, então, impulsionadora do processo de refletir-experienciar-transformar, estratégia fundamental para mudar a cultura do cuidado ritualizado.

Cuidar e pesquisar tornam-se um binômio que demanda competência e esforço da enfermeira. Faz-se necessária, portanto, a introdução de estratégias de apoio e mediação, por meio da educação permanente, que favoreçam a sua efetivação no contexto das instituições de saúde.

Sobre a mediação no processo investigativo, Dyniewicz (2003) enfatiza que a pesquisa deve ser considerada como habilidade a ser incentivada para ser desenvolvida e não apenas como atividade inerente e natural aos enfermeiros.

O incentivo possibilitado pelo NUPE, associado aos esforços dos enfermeiros e da instituição, tem resultados da produção científica das enfermeiras assistenciais a serem descritos e analisados.

\section{Objetivo}

Frente a este cenário organizacional, realizamos o estudo com o objetivo de Analisar as publicações científicas de enfermeiras assistenciais do HURNP, a partir da implantação do NUPE, enfocando a produção investigativa dos anos de 1999 a 2002.

\section{Metodologia}

O estudo foi realizado no Hospital Universitário Regional do Norte do Paraná (HURNP), órgão suplementar da Universidade Estadual de Londrina (UEL), o qual é vinculado administrativamente à Reitoria e academicamente ao Centro de Ciências da Saúde (CCS).

Para responder ao objetivo delineado, buscamos, a partir da implantação do NUPE, os resultados obtidos com a produção científica das enfermeiras. Os dados foram levantados por meio de pesquisa documental nos relatórios mensais do NUPE e na Assessoria Técnica e de Planejamento de Recursos Humanos da Diretoria de Enfermagem. As informações foram confrontadas com relatos de outros estudos, obtidos na literatura da área.

Em um primeiro momento, descrevemos os dados quantitativos, apresentados sob a forma de figura e relativos aos artigos científicos publicados.

Na seqüência, analisamos a direcionalidade dos temas desenvolvidos nos artigos publicados, tomando como referência o esquema categorial proposto por Carvalho (2000) e referendado no Programa Nacional de Pós-Graduação (PNPG) da CAPES, a fim de agrupar a produção publicada pelas enfermeiras assistenciais no período de 1999 a 2002. O esquema apresenta três categorias ou campos temáticos, a saber: Profissional, Assistencial e Organizacional.

Segundo Gutiérrez et al. (2001), essas categorias dizem respeito às questões relacionadas à profissão (profissional), aos estudos centrados na clientela (assistencial), e ao que se refere à organização de serviços de saúde e de enfermagem (organizacional).

Para tanto, resgatamos todos os artigos publicados e procedemos à leitura analítica a fim de realizar a categorização conforme os esquemas prédefinidos, além de levantarmos informações sobre a autoria e fonte da publicação dos mesmos. Os trabalhos premiados no período também foram identificados. 


\section{Resultados e Discussão}

Anteriormente ao início das atividades do NUPE, a atividade investigativa, por parte das enfermeiras dessa instituição, era, no geral, inexpressiva e inconstante. Esta situação evidenciava a cultura organizacional até então, pela inexistência de estratégias de incentivo por parte da organização e a não motivação das enfermeiras para a atividade.

Deter-nos-emos na análise dos artigos publicados e neste sentido evidenciamos na Figura 1 a evolução das publicações no período.

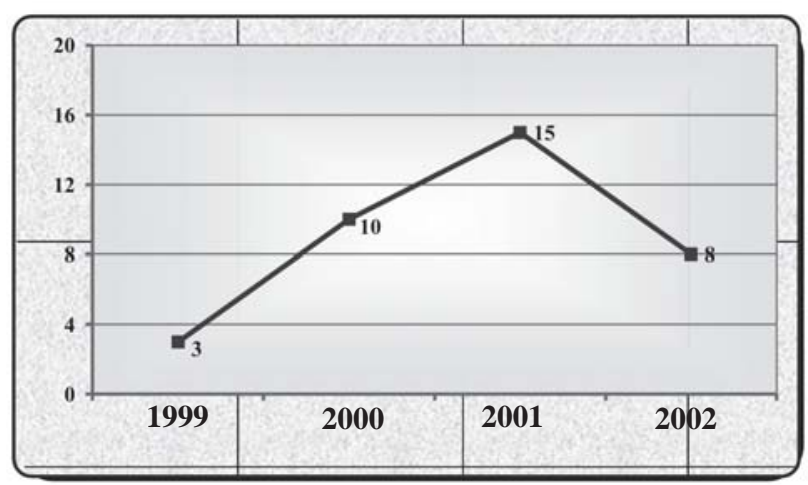

Figura 1. Artigos científicos publicados por enfermeiras assistenciais do HURNP nos anos de 1999 a 2002, Londrina-Pr 2003.

Verificamos que, nos anos de 1999 a 2001, a linha do número de publicações de artigos foi ascendente e, em 2002, descendente. A explicação para tal fato está diretamente associada ao momento vicenciado na Instituição com as eleições e posse das Diretorias do HURNP, situação que suscitou, entre as enfermeiras, expectativas e desaceleração nas atividades enquanto aguardavam as novas diretrizes políticas para a gestão 2002-2006.

Salzano et al. (1998) verificaram, em estudo realizado entre enfermeiros de instituições públicas e privadas, que os profissionais de hospitais privados publicam mais, possivelmente por melhores condições de trabalho existentes na instituição e a um menor percentual de profissionais com duplo vínculo empregatício. Isso, certamente, representa um fator estimulador do incremento da produção, junto com outras medidas de incentivo.
Adami et al. (1996), em levantamento acerca da situação da pesquisa em enfermagem, realizado em hospitais do Município de São Paulo- SP, identificaram, num período de cinco anos, de quarenta trabalhos produzidos em sete hospitais privados, dos quais apenas dezessete foram publicados.

Esses autores, na continuidade do estudo, ampliaram a análise para seis anos e meio e verificaram que a produção cientifica dos mesmos hospitais, com a exceção de um deles, deu um salto qualitativo, em apenas um ano e meio. A média de trabalho por enfermeiro assistencial correspondente aos dois períodos analisados subiu de 0,03 para 0,32 trabalhos, ou seja, de 0,006 para 0,05 trabalho por enfermeiro/ano. $\mathrm{O}$ número de apresentações em eventos científicos, bem como nas publicações, elevaram-se, denotando um maior envolvimento das enfermeiras assistênciais no desenvolvimento de pesquisas.

Costa e Carvalho (2001) em estudo sobre a produção científica dos enfermeiros do Estado de Minas Gerais, publicada em periódicos de enfermagem nacionais que compõem o Banco de Dados de Enfermagem-BDENF, averiguaram que, dos 53 autores de trabalhos científicos, apenas 10 (18\%) são de enfermeiros assistenciais, o que representa uma produção ainda sensivelmente pequena desta categoria profissional.

Dyniewicz (2003), após dois anos de desenvolvimento de práticas educativas em metodologia de pesquisa, em hospital universitário em Curitiba-PR., verificou a publicação de 14 artigos elaborados por 28 enfermeiras. Foi alcançanda, portanto, uma média de 0,5 por enfermeira para o período e uma média de 0,25 publicações por enfermeira/ano.

Leão (2005b) notou que a produção científica das enfermeiras assistenciais, trabalhadoras de um serviço de saúde privado na cidade de São PauloSP, vem aumentando de forma paulatina mas constante, sendo a assessoria de pesquisa um recurso facilitador do engajamento das profissionais, 
que publicaram, ao longo de três anos consecutivos, 32 artigos em periódicos e capítulos de livros. Considerando-se somente os profissionais envolvidos na produção científica publicada, o total equivale a $33 \%$ do quadro institucional (43 enfermeiras), sendo a média de 0,74 para o período de estudo, por enfermeira, e a média de 0,24 publicações, por enfermeiro/ano.

Comparando a produção científica publicada pelas enfermeiras assistenciais assistidas pelo NUPE com os estudos de Dyniewicz (2003) e Leão (2005b), verificamos que 36 artigos foram produzidos por 23 enfermeiras ( $25 \%$ da média do quadro funcional/ano), com co-autoria de outros profissionais e alunos, como veremos a seguir. Isso perfaz a média de 1,56 artigos por enfermeira e 0,39 publicações por enfermeira/ano. Sendo assim, a produção das enfermeiras do HURNP foi maior que a das outras instituições alvos dos outros dois estudos. Podemos inferir que quanto maior o tempo de atividades do serviço de assessoria em pesquisa junto às enfermeiras, maior a participação delas nas atividades investigativas e, conseqüentemente, maiores possibilidades de publicações.

Vale lembrar a reflexão de Leão (2005b, p.86) acerca do cálculo de indicadores, quando explicita que estes servem mais como instrumento de análise e acompanhamento institucional interno, uma vez que "o estabelecimento do benchmarking externo (técnica de comparação entre metas, fatos, dados e informações pautadas em parâmetros internos e externos, que permite conhecer as mudanças ocorridas na instituição), enfrenta diversas barreiras". O autor, em suas reflexões, cita as muitas diferenças entre os serviços hospitalares, os quais ainda são poucos acompanhados por uma assessoria específica da área, além da pouca literatura acerca do tema.

Sendo assim, compartilhamos da opinião de Leão (2005b) quando diz que os indicadores, examinados na perspectiva da frieza dos números, mostram que é expressivamente baixa a produção científica dos enfermeiros assistenciais.
Verifica-se que os indicadores apontam resultados de uma realidade que ainda está longe do que almejamos e não refletem a magnitude dos trabalhos e publicações realizados pelas enfermeiras. Faz-se necessária, pois, uma reflexão mais pormenorizada acerca dessa produção, de modo que se explores e valorize a construção do conhecimento elaborado por enfermeiras assistenciais.

Costa e Carvalho (2001) enfatizam que a divulgação é importante em qualquer área profissional e, evidentemente, também na enfermagem, pois as novas informações podem promover novas aprendizagens.

Ressaltam, também, que "a comunicação dos resultados da pesquisa permite que outros profissionais tenham a oportunidade de conhecer, avaliar e questionar problemas que eventualmente possam surgir sobre as questões estudadas" (COSTA; CARVALHO, 2001, p.20).

Nesse sentido, passamos a descrever nas Tabelas e Figuras, na sequência, a análise realizada sobre os trabalhos publicados, enfocando as características referentes à autoria, ao veículo de divulgação, às categorias e à classificação dos artigos em relação às indicações bibliográficas.

A Tabela 1 apresenta a distribuição dos artigos publicados pelas enfermeiras, no período do estudo, por número de autores.

Tabela 1. Distribuição dos artigos publicados pelas enfermeiras assistenciais do HURNP, no período de 1999-2002, por número de autores, Londrina-Pr, 2003.

\begin{tabular}{ccc}
\hline \multirow{2}{*}{$\begin{array}{c}\text { NoutORES POR } \\
\text { ARTIGO }\end{array}$} & \multicolumn{2}{c}{ ARTigos } \\
\cline { 2 - 3 } 1 & 6 & $\%$ \\
\hline 2 & 10 & 16,6 \\
3 & 10 & 27,7 \\
4 & 4 & 27,7 \\
5 & 4 & 11,1 \\
6 & 2 & 11,1 \\
\hline TOTAL & 36 & $\mathbf{1 0 0 , 0}$ \\
\hline
\end{tabular}

* Aproximado para $100,0 \%$ 
Na Tabela 1, verificamos que dos 36 artigos publicados, $30(83,3 \%)$ são de autoria múltipla. A participação de vários profissionais na elaboração de um artigo é uma peculiaridade divulgada nos meios científicos, visando reunir, numa mesma tarefa, as forças, conhecimentos e responsabilidades de interessados em tema afim. A autoria múltipla, segundo Mendes (1991), denota valorização do trabalho em grupo.

A Tabela 2 apresenta a produção de conhecimento no período do estudo, segundo a categoria dos autores.

Tabela 2. Distribuição da produção de conhecimento no período de 1999 a 2002, segundo a categoria dos autores, Londrina-Pr, 2003.

\begin{tabular}{lcc}
\hline \multicolumn{1}{c}{ CATEgoria / AGRuPamento } & $\mathrm{f}$ & $\%$ \\
\hline Enfermeiras & 16 & 44,4 \\
Enfermeira e aluno de enfermagem & 0 & 0,0 \\
Enfermeira, aluno e docente de enfermagem & 2 & 5,6 \\
Enfermeira e professor de enfermagem & 5 & 139 \\
Enfermeira e profissionais de saúde & 12 & 33,4 \\
Enfermeira, docente de enfermagem e outros profissionais & 1 & 2,7 \\
\hline TOTAL & $\mathbf{3 6}$ & $\mathbf{1 0 0 , 0}$ \\
\hline
\end{tabular}

Na Tabela 2, observamos que $16(44,4 \%)$ artigos foram desenvolvidos apenas por enfermeiras; $12(33,4 \%)$, por enfermeiras e outros profissionais da saúde; $5(13,9 \%)$ por enfermeiras e docentes de enfermagem; $2(5,6 \%)$ por enfermeiras, docentes e alunos de enfermagem; e apenas $1(2,7 \%)$ por enfermeira, docente e outros profissionais.

Nesse grupo avaliamos que na fase inicial dos trabalhos investigativos, foi natural o agrupamento das próprias profissionais. Contudo, a atuação em equipe multiprofissional também foi observada, num índice expressivo, como ocorreu nos artigos que envolviam enfermeiras e docentes. O agrupamento de enfermeiras, docentes e alunos aconteceu em menor proporção, e o mesmo se deu entre enfermeiro, docente e outros profissionais.

Costa e Carvalho (2001) consideram que existe uma tendência para a realização de trabalhos em grupo e ressaltam que este fato é importante para o desenvolvimento da produção de conhecimento em Enfermagem.

A divulgação dos resultados da pesquisa em enfermagem, pela publicação em periódicos, facilita o acesso dos pares para avaliar as contribuições feitas pelos indivíduos e instituições acadêmicas na área de conhecimento (COSTA; CARVALHO, 2001; CALIRI, 2002).

Partilhamos dessa opinião e verificamos que o NUPE incentivou as enfermeiras no intuito de difundir amplamente os conhecimentos e experiências, publicando-os em periódicos, jornais, informativos editados e on-line, como podemos verificar na Tabela 3.

Tabela 3. Distribuição dos artigos publicados por enfermeiras assistenciais do HURNP, segundo o Veiculo de Divulgação, 19992002, Londrina, 2003.

\begin{tabular}{lcr}
\hline \multirow{2}{*}{ VEíCulos DE DIVULGAÇÃo } & \multicolumn{2}{c}{$\mathbf{N}^{*}$ DE ARTIGOS } \\
\cline { 2 - 3 } & $\mathrm{f}$ & $\%$ \\
\hline Informativo Sobest & 1 & 2,7 \\
Revista Espaço para Saúde & 7 & 19,4 \\
Unopar Cientifica - Ciências Biológica e Saúde & 4 & 11,1 \\
Revista Latino-americana de Enfermagem** & 2 & 5,5 \\
Nursing** & 5 & 13,8 \\
OLHO MÁGICO** & 5 & 13,8 \\
Revista Diabetes Clínica & 1 & 2,7 \\
Revista Higiene Alimentar** & 1 & 2,7 \\
Revista de Cirurgia Infantil & 1 & 2,7 \\
Arquivos de Ciências da Saúde - Unipar** & 1 & 2,7 \\
Revista Escola de Enfermagem - USP** & 1 & 2,7 \\
Jornal Paranaense de Pediatria & 1 & 2,7 \\
SEMINA - Ciências Biológicas e de Saúde** & 2 & 5,5 \\
Revista Cogitare** & 4 & 11,1 \\
\hline Total & 36 & $\mathbf{1 0 0 , 0 *}$ \\
\hline * Aproximado para 100,0\% - ** Periódicos & indexados \\
\hline
\end{tabular}

A distribuição dos artigos publicados, apresentada na Tabela 3, demonstra uma gama variada de veículos de divulgação do conhecimento produzido na área da saúde/enfermagem. Observamos que, dos 14 veículos de divulgação, 08 são indexados em alguma base de dados e os demais são periódicos que não fazem referências a essa informação. Existe a possibilidade desses veículos de divulgação estarem na fase de solicitação 
e tramitando pela burocracia da Indexação em base de dados, aspecto essencial no tocante à credibilidade do periódico e às informações divulgadas.

Rosas (2001) esclarece que para acontecer a transferência da informação, istoé, a divulgação dos novos conhecimentos, os cuidados começam com a redação do trabalho, passam pela escolha do periódico para publicação e vão até a indexação em base de dados.

Secaf (2000) orienta sobre a produção e divulgação de artigo científico, dizendo ser importante que se dê atenção especial à elaboração do título e à escolha das palavras-chave, pois este conjunto de informações propiciará a devida indexação e, assim, constituirá uma forma fácil e correta de recuperar a informação desejada. Diz ainda que, para os profissionais da área da saúde, as palavras-chave são extraídas dos Descritores em Ciências da Saúde (DCS) e do Centro LatinoAmericano e do Caribe de Informação em Ciências da Saúde (BIREME).

Em relação à indexação dos periódicos, segundo a classificação do QUALIS da CAPES, os veículos de divulgação utilizados para as publicações são classificados entre C-Local (Olho Mágico), CNacional (Revista Espaço para Saúde; Revista Diabetes Clínica, Revista Higiene Alimentar; Arquivos de Ciências da Saúde - Unipar; Jornal Paranaense de Pediatria), B-Nacional (SEMINA Ciências Biológicas e da Saúde, Revisa Cogitare, Revista de Cirurgia Infantil, Nursing), CInternacional (Revista Escola de Enfermagem USP) e B-Internacional (Revista Latino-Americana de Enfermagem).

A classificação do QUALIS da CAPES fornece um indicador de qualidade e do âmbito de circulação dos veículos sistematicamente utilizados para a divulgação de trabalhos técnicos e científicos dos cursos de pós-graduação. É um importante indicador da qualidade da produção dos veículos de divulgação e uma referência importante na escolha e determinação do periódico para a divulgação dos resultados de pesquisa.

Salientamos que a divulgação formal, isto é, a publicação de artigo científicoé o meio de divulgação que oferece credibilidade suficiente para que a informação sirva de suporte para outras pesquisas. Mas não basta publicar em qualquer veículo de divulgação: é preciso que o texto seja divulgado amplamente e por periódico aceito no meio científico e, assim, possa ser lido, aceito e utilizado (MANCIA; RAMOS, 2002).

A orientação acerca dos quesitos relacionados à Indexação e ao QUALIS da CAPES às enfermeiras, autoras de artigos, que pretendam publicá-los, deve ser aspecto a ser lembrado, porquanto esse cuidado é básico para que alcancem uma ampla divulgação de seus trabalhos e, dessa forma, possam colaborar efetivamente para o avanço da enfermagem. Observamos, porém, que a escolha do periódico, muitas vezes, se faz mediante o valor da assinatura, ou até, caso esta não seja necessária, à possibilidade de uma rápida publicação, critérios tidos como mais decisivos do que a própria importância e alcance do veículo de divulgação. São paradigmas a serem esclarecidos para se poder ultrapassá-los.

Na Tabela 4, observamos que as Linhas $2.1 \mathrm{e}$ 3.4, na distribuição anual da produção científica referente ao campo temático Assistencial e Organizacional, respectivamente, mantiveram-se constantes em todos os anos. Esta configuração confirma a preocupação das enfermeiras, ao realizarem atividades investigativas sobre temáticas relacionadas ao processo de cuidar e gerenciar em saúde e enfermagem. Essa tendência natural decorre das necessidades experimentadas por quem vive $\mathrm{e}$ trabalha a assistência no dia-a-dia. 
Tabela 4. Distribuição dos artigos publicados por enfermeiras assistenciais do HURNP, segundo o campo temático, linha de pesquisa, 1999 a 2002, referendado no Programa Nacional de pósgraduação (PNPG) da CAPES, Londrina- Pr, 2003.

\begin{tabular}{|c|c|c|c|c|c|c|}
\hline \multirow{2}{*}{ САMPO ТЕмÁTICO } & \multirow{2}{*}{ LINHA } & \multicolumn{4}{|c|}{ AnOs } & \multirow{2}{*}{ TOTAL } \\
\hline & & 1999 & 2000 & 2001 & 2002 & \\
\hline \multirow{3}{*}{ Assistencial } & $\begin{array}{l}\text { 2.1 Processo de Cuidar em } \\
\text { Saúde e Enfermagem }\end{array}$ & 1 & 4 & 4 & 3 & 12 \\
\hline & & & & & & \\
\hline & 2.2 Saúde e Qualidade de Vida & 0 & 1 & 1 & 0 & 2 \\
\hline \multirow{3}{*}{ Organizacional } & $\begin{array}{l}\text { 3.2 Políticas e Práticas de } \\
\text { Educação e Enfermagem }\end{array}$ & 0 & 2 & 5 & 0 & 7 \\
\hline & $\begin{array}{l}\text { 3.3 Produção social e Trabalho } \\
\text { em Saúde e Enfermagem }\end{array}$ & 0 & 0 & 2 & 3 & 5 \\
\hline & $\begin{array}{l}3.4 \text { Gerenciamento dos } \\
\text { Serviços de Saúde e de } \\
\text { Enfermagem }\end{array}$ & 2 & 3 & 3 & 2 & 10 \\
\hline
\end{tabular}

Cabe salientar que a participação das enfermeiras se faz ainda mais decisiva, junto aos demais atores sociais da saúde para a melhoria dos serviços de saúde prestados à população, mediante acompanhamento dos movimentos e propostas elencados como Prioridades de Pesquisa da Agência Nacional de Pesquisa em Saúde, do Ministério da Saúde do Brasil. Nesse sentido, Marziale (2004, p.578) argumenta que as enfermeiras devem "reconhecer a importância da contribuição dos resultados da pesquisa à prática profissional e incrementar a produção de suas pesquisas em sintonia com as diretrizes traçadas pelos organismos nacionais e internacionais e agências de apoio à pesquisa".

A Agenda Nacional de Prioridades de Pesquisa em Saúde tem como meta aumentar a capacidade de indução de pesquisas voltadas para melhorar as condições de saúde da população. A referida Agenda permite estabelecer prioridades para pesquisas que abranjam as áreas de conhecimento relacionadas à saúde, à pesquisa básica, à clínica e metodológica, capazes de melhorar as práticas, procedimentos, equipamentos, serviços, programas e políticas de saúde (BRASIL, 2004).

Agudelo (2004), ao referir sobre as tendências e prioridades em investigação em Enfermagem, faz algumas reflexões acerca do processo de definição de prioridades na produção de conhecimento da enfermagem, e ressalta ser importante levar em consideração as necessidades individuais e coletivas de saúde, na saúde coletiva e clínica. Dessa maneira, esse poderá responder tanto às necessidades de fundamentação das disciplinas, como às necessidades de saúde das populações, realizadas em diferentes abordagens teóricas e metodológicas.

Essas diretrizes são políticas e recomendações necessárias para que tomemos posição acertada quanto às prioridades em pesquisa e não fiquemos insistindo em temáticas amplamente investigadas: antes faz-se necessário replicar pesquisas acerca de nossas realidades, para analisarmos o que é melhor fazer, para um melhor cuidado com a saúde. Contudo, o contexto clínico-assistencial está imerso em necessidades especificas para o alcance de um 
atendimento digno, repleto de situações complexas, e clama por soluções imediatistas e, muitas vezes, distante do que dispõe a agenda nacional e internacional. Isso nos leva a concluir que temos um longo caminho a percorrer no sentido de contribuir para os avanços na área da saúde, direcionando nossas pesquisas ao que é prioritário.

$\mathrm{Na}$ seqüência apresentamos, em forma de Quadros, a distribuição das publicações referentes às Linhas/Campo Temático, descritos no início do texto. Os artigos são também descritos segundo a autoria, título, veículo de divulgação e demais indicacões bibliográficas.
Informamos que a Figura 2 refere-se à Linha 2.1 - Processo de Cuidar em Saúde e Enfermagem; A Figura 3, à Linha 2.2 - Saúde e Qualidade de Vida, ambos referentes ao campo temático Assistencial; a Figura 4, refere-se à Linha 3.2 - Políticas e Práticas de Educação e Enfermagem; a Figura 5, à Linha 3.3- Produção Social e Trabalho em Saúde e Enfermagem; e a Figura 6, à Linha 3.4 Gerenciamento dos Serviços de Saúde e de Enfermagem, relacionadas, ao campo temático Organizacional.

\begin{tabular}{|c|c|c|c|}
\hline Autores & Título & $\begin{array}{l}\text { VEÍCULO DE } \\
\text { DIVULGAÇÃO }\end{array}$ & INDICAÇÕES \\
\hline R.C. Domansky & Incontinência Anal: Revisão Bibliográfica & Informativo Sobest & $\begin{array}{c}\text { ano I, ed.III, } \\
1999\end{array}$ \\
\hline $\begin{array}{l}\text { M.C.L. Haddad } \\
\text { L.C. Bruschi } \\
\text { E.A.P. Martins }\end{array}$ & $\begin{array}{l}\text { Influência do açúcar no processo de cicatrizaçã } \\
\text { de incisões cirúrgicas infectadas }\end{array}$ & $\begin{array}{l}\text { evista Latino-Americana } \\
\text { de Enfermagem }\end{array}$ & $\begin{array}{l}\text { v.8, n. } 1, \\
\text { p. } 57-65, j \\
\text { an } / 2000\end{array}$ \\
\hline $\begin{array}{l}\text { E.A.P. Martins } \\
\text { M.C.L. Haddad }\end{array}$ & $\begin{array}{l}\text { Validação de um instrumento que classifica } \\
\text { os pacientes em quatro graus de dependência } \\
\text { do cuidado de enfermagem }\end{array}$ & $\begin{array}{l}\text { evista Latino-Americana } \\
\text { de Enfermagem }\end{array}$ & $\begin{array}{l}\text { v. } 8, \text { n. } 2 \text {, } \\
\text { p. } 74-82 \text {, } \\
\text { abr/ } 2000\end{array}$ \\
\hline $\begin{array}{l}\text { M.T. Parra } \\
\text { A.E.P. Moraes } \\
\text { R.C. Domansky }\end{array}$ & $\begin{array}{l}\text { Alta Hospitalar: A Família e o } \\
\text { Cuidado no Domicílio }\end{array}$ & OLHO MÁGICO & $\begin{array}{l}\text { v. } 6, \mathrm{n} .22 \\
\text { p. } 19-22 \\
\text { ago/2000 }\end{array}$ \\
\hline $\begin{array}{l}\text { E.A.P. Martins } \\
\text { M.C.L. Haddad } \\
\text { I.A. Secco }\end{array}$ & Curativos: Compartilhando as Inovações & $\begin{array}{l}\text { Unopar Cientifica - } \\
\text { Ciências biológicas e } \\
\text { da Saúde }\end{array}$ & $\begin{array}{l}\text { v. } 2, \mathrm{n} .1 \text {, } \\
\text { out } / 2000\end{array}$ \\
\hline $\begin{array}{l}\text { I. Popper } \\
\text { M.M.B. Oliveira } \\
\text { M.T.O. Vannuchi }\end{array}$ & $\begin{array}{l}\text { Produção de Leite Humano Ordenhado "Zero } \\
\text { Defeitos": Uma proposta }\end{array}$ & evista Higiene Alimentar & $\begin{array}{c}\mathrm{n} .84, \mathrm{n} .15 \\
\mathrm{mai} / 2001\end{array}$ \\
\hline $\begin{array}{l}\text { C.B. Soares } \\
\text { M.G.P. Cardoso }\end{array}$ & $\begin{array}{l}\text { Metodologia da Assistência de Enfermagem } \\
\text { na Unidade de Tratamento Dialítico }\end{array}$ & $\begin{array}{l}\text { Arquivos de Ciências } \\
\text { da Saúde da Unopar }\end{array}$ & $\begin{array}{c}\text { v.5, n.3, } \\
\text { set-dez/2001 }\end{array}$ \\
\hline $\begin{array}{l}\text { C.B. Soares } \\
\text { E.Y. Ochiro } \\
\text { N.T. Sannomiya }\end{array}$ & $\begin{array}{c}\text { Relação da Temperatura da Solução de Diálise } \\
\text { e a Hipotensão Arterial Sintomática Observada } \\
\text { durante sessões de hemodiálise em Pacientes } \\
\text { com Insuficiência Renal Crônica }\end{array}$ & $\begin{array}{l}\text { Revista Escola de } \\
\text { Enfermagem USP }\end{array}$ & $\begin{array}{l}\text { v. } 35, \mathrm{n} .4, \\
\text { p. } 346-53,2001 \\
\text { (novembro) }\end{array}$ \\
\hline C.B. Godoy & Atendimento de Enfermagem em puericultura & $\begin{array}{l}\text { Jornal Paranaense de } \\
\text { Pediatria }\end{array}$ & $\begin{array}{l}\text { v. } 2, \mathrm{n} .4, \mathrm{p} .40 \\
2001\end{array}$ \\
\hline $\begin{array}{l}\text { S.K.S. Barros } \\
\text { M.C.L. Haddad } \\
\text { E.H.T. Anam; } \\
\text { M.H.D.M. Guarient }\end{array}$ & $\begin{array}{c}\text { Aplicação de protocolo para prevenção } \\
\text { de úlcera de pressão em Unidade } \\
\text { de Terapia Intensiva }\end{array}$ & $\begin{array}{l}\text { Revista Semina Ciências } \\
\text { Biológicas e de Saúde }\end{array}$ & $\begin{array}{l}\text { v. } 23 \\
2002\end{array}$ \\
\hline
\end{tabular}


M.H.D.M. Guarient

M.E. Karino

M.C.L. Haddad

Adolescentes com Diabetes Mellitus: vantagens e limites da monitorização

F. Kasai

C. Faria

I R S Freitas

M.H.D.M. Guarient

M.E. Karino

M.C.L. Haddad

F. Kasai

C. Faria

I.R.S. Freitas

$\begin{array}{cc}\text { Revista Cogitare } & \text { v.7, n. } 1, \\ \text { Enfermagem } & \text { p.48-54 } \\ & 2002\end{array}$

$\begin{array}{cc}\text { Unopar Cientifica - } & \text { v.1, n.1, } \\ \text { Ciências biológicas } & \text { p.7-19, } \\ \text { e da Saúde } & 2002\end{array}$

Figura 2. Distribuição das publicações realizadas por enfermeiras assistenciais do HURNP na Linha de Pesquisa Processo de Cuidar em Saúde e Enfermagem, segundo autoria, título, veículo de divulgação e demais Indicações Bibliográficas, 1999-2002, Londrina-Pr, 2003.

\begin{tabular}{|c|c|c|c|}
\hline Autores & Título & $\begin{array}{l}\text { VEÍCULO dE } \\
\text { DIVULGAÇÃO }\end{array}$ & INDICAÇÕES \\
\hline $\begin{array}{l}\text { B.N. Teodoro } \\
\text { S.M. Andrade }\end{array}$ & $\begin{array}{l}\text { Internações Pediátricas no Hospital } \\
\text { Universitário Regional do Norte do Paraná }\end{array}$ & $\begin{array}{l}\text { Revista Espaço } \\
\text { para a Saúde }\end{array}$ & $\begin{array}{l}\text { on-line v.1, n.2, } \\
\text { p.89-111, jun/2000 }\end{array}$ \\
\hline $\begin{array}{l}\text { I.T. Takahashi } \\
\text { H.G. Almeida } \\
\text { M.H.D.M. Guariente } \\
\text { I. Staurt } \\
\text { M.C.L. Haddad }\end{array}$ & $\begin{array}{l}\text { Perfil dos Diabéticos Assistidos em duas } \\
\text { Unidades Básicas de Saúde de Londrina - } \\
\text { Pr }\end{array}$ & $\begin{array}{l}\text { Revista Diabetes } \\
\text { Clínica }\end{array}$ & $\begin{array}{l}\text { n.2, p. } 122-28 \\
\text { mar/abr } 2001 \\
\quad \text { S.Paulo }\end{array}$ \\
\hline
\end{tabular}

Figura 3. Distribuição das Publicações realizadas por enfermeiras assistenciais do HURNP na Linha de Pesquisa Saúde e Qualidade de Vida, segundo autoria, título, veículo de divulgação e demais Indicações bibliográficas, 1999-2002, Londrina-Pr, 2003.

\begin{tabular}{|c|c|c|c|}
\hline AUTORES & Título & $\begin{array}{l}\text { VEICULO DE } \\
\text { DIVULGAÇÃO }\end{array}$ & INDICACOES \\
\hline $\begin{array}{l}\text { M.V. Fernandes } \\
\text { R. Antunes } \\
\text { M.B. Camuci } \\
\text { M.H.D.M. Guariente }\end{array}$ & $\begin{array}{l}\text { Mudança de Comportamento: em } \\
\text { desafio frente à infeç̧ão hospitalar }\end{array}$ & OLHO MÁGICO & $\begin{array}{l}\text { v. } 6, \text { n. } 21 \\
\text { p. } 20-22 \\
\text { maio/2000 }\end{array}$ \\
\hline $\begin{array}{l}\text { M. Karino } \\
\text { M.H.D.M. Guariente }\end{array}$ & $\begin{array}{l}\text { O aprendizado do aluno no primeiro estágio } \\
\text { de enfermagem: a visão do aluno }\end{array}$ & $\begin{array}{c}\text { Unopar Cientifica } \\
\text { Ciências Biológicas } \\
\text { e da Saúde }\end{array}$ & $\begin{array}{l}\text { v.5, n.1, } \\
\text { p.33-34, } 2001\end{array}$ \\
\hline $\begin{array}{l}\text { M.H.D.M. Guariente } \\
\text { N.A.N. Berbel } \\
\text { Z. Soubhia }\end{array}$ & Avaliação: Uma questão sempre atual & OLHO MÁGICO & $\begin{array}{c}\text { v. } 8, \mathrm{n} .1 \\
\text { p. } 34-36, \text { jan/abr } \\
2001\end{array}$ \\
\hline $\begin{array}{l}\text { R.A. Belei } \\
\text { F. Oliveira } \\
\text { N. Paiva } \\
\text { E. Cavassin }\end{array}$ & $\begin{array}{c}\text { Estágio Voluntário na Comissão de Controle } \\
\text { de Infecção Hospitalar: inovações nas } \\
\text { estratégias educacionais }\end{array}$ & OLHO MÁGICO & $\begin{array}{l}\text { v.8, n.2, p.25-26 } \\
\text { mai/ago } 2001\end{array}$ \\
\hline $\begin{array}{l}\text { M.C.L. Haddad } \\
\text { I.T. Martins }\end{array}$ & Um novo olhar sobre a educação & OLHO MÁGICO & $\begin{array}{l}\text { v.8, n.2, p.25 -26 } \\
\text { mai/ago } 2001\end{array}$ \\
\hline C.B. Godoy & $\begin{array}{l}\text { Educação Sexual para Adolescentes: } \\
\text { uma experiência para a enfermagem }\end{array}$ & $\begin{array}{l}\text { Jornal Paranaense } \\
\text { de Pediatria }\end{array}$ & $\begin{array}{l}\text { v. } 2, \text { n. } 4, \mathrm{p} .40 \\
2001\end{array}$ \\
\hline $\begin{array}{l}\text { M.C.L. Haddad } \\
\text { M.H.D.M. Guariente }\end{array}$ & $\begin{array}{l}\text { A inserção do Método Cientifico na prática e } \\
\text { na reflexão dos enfermeiros do campo }\end{array}$ & Nursing & $\begin{array}{l}\text { v. } 3, \text { n.27, p. } 16- \\
18, \text { ago/2000 }\end{array}$ \\
\hline
\end{tabular}

Figura 4 - Distribuição das Publicações realizadas por enfermeiras assistenciais do HURNP na Linha de Pesquisa Políticas e Práticas de Educação e Enfermagem, segundo autoria, título, veículo de divulgação e demais Indicações Bibliográficas, 1999-2002, Londrina-Pr, 2003. 


\begin{tabular}{|c|c|c|c|}
\hline AUTORES & Título & $\begin{array}{l}\text { Veiculo de } \\
\text { Divulgação } \\
\end{array}$ & INDICAÇÕES \\
\hline $\begin{array}{l}\text { D. R. Costa } \\
\text { L.T. Marchese et al. }\end{array}$ & $\begin{array}{l}\text { Acidentes Inadvertidos com } \\
\text { equipe cirúrgica }\end{array}$ & $\begin{array}{l}\text { Revista de Cirurgia } \\
\text { Infantil }\end{array}$ & v.10, 2001 \\
\hline $\begin{array}{l}\text { R.A. Belei } \\
\text { C.M. Carrilho } \\
\text { N. Paiva } \\
\text { D. Geani } \\
\text { F. Oliveira } \\
\text { M.H.D.M. Guariente }\end{array}$ & $\begin{array}{l}\text { O impacto do acidente com material } \\
\text { biológico na vida de profissionais } \\
\text { e alunos de um hospital universitário }\end{array}$ & $\begin{array}{l}\text { Revista Espaço } \\
\text { para Saúde }\end{array}$ & $\begin{array}{l}\text { v.2, n.2, } \\
\text { jun/2001 }\end{array}$ \\
\hline $\begin{array}{l}\text { A.D.D. Sentone } \\
\text { A. A. F. Gonçalves }\end{array}$ & $\begin{array}{l}\text { O sofrimento do trabalhador com } \\
\text { dois vínculos empregatícios }\end{array}$ & $\begin{array}{l}\text { Ciências Biológicas } \\
\text { e da Saúde }\end{array}$ & $\begin{array}{l}\text { on-line, v.23, } \\
2002\end{array}$ \\
\hline $\begin{array}{l}\text { I.A. Secco } \\
\text { P.R. Gutierrez } \\
\text { T. Matsuo } \\
\text { M.L. Robazzi }\end{array}$ & $\begin{array}{l}\text { A equipe de enfermagem de } \\
\text { hospital público e os acidentes de } \\
\text { trabalho com material biológico }\end{array}$ & $\begin{array}{c}\text { Revista Semina } \\
\text { Ciências Biológicas } \\
\text { e da Saúde }\end{array}$ & $\begin{array}{c}\text { on-line, v.23, } \\
2002\end{array}$ \\
\hline $\begin{array}{l}\text { I.A. Secco } \\
\text { P.R. Gutierrez } \\
\text { T. Matsuo } \\
\text { M.L. Robazzi }\end{array}$ & $\begin{array}{l}\text { Acidentes de Trabalho em } \\
\text { ambiente hospitalar }\end{array}$ & $\begin{array}{c}\text { Revista Semina } \\
\text { Ciências Biológicas } \\
\text { e da Saúde }\end{array}$ & $\begin{array}{l}\text { p. } 19-23 \text {, } \\
\text { nov/2002 }\end{array}$ \\
\hline
\end{tabular}

Figura 5. Distribuição das publicações realizadas por enfermeiras assistenciais do HURNP na Linha de Pesquisa Produção Social e Trabalho em Saúde e Enfermagem, segundo autoria, título, veículo de divulgação e demais indicações bibliográficas, 1999-2002, Londrina-Pr, 2003.

\begin{tabular}{|c|c|c|c|}
\hline AUTORES & TítuLo & $\begin{array}{l}\text { VEICULO DE } \\
\text { DIVULGACÃO }\end{array}$ & INDICACCÕES \\
\hline $\begin{array}{l}\text { R.A. Belei } \\
\text { M.S.Tavares } \\
\text { N.S. Paiva }\end{array}$ & $\begin{array}{c}\text { Lixo Hospitalar: viabilidade econômica } \\
\text { de uma Fonte Alternativa de Recursos } \\
\text { em um Hospital Universitário }\end{array}$ & $\begin{array}{l}\text { Revista Espaço } \\
\text { para Saúde }\end{array}$ & $\begin{array}{c}\text { v. } 1, \text { n. } 1, \text { p. } 48-64 \\
1999\end{array}$ \\
\hline $\begin{array}{l}\text { R.A. Belei } \\
\text { M.S.Tavares } \\
\text { N.S. Paiva }\end{array}$ & Lixo e Serviços de Saúde: uma revisão & $\begin{array}{l}\text { Revista Espaço } \\
\text { para Saúde }\end{array}$ & $\begin{array}{c}\text { v. } 1, \text { n. } 1, \text { p. } 25-47 \\
1999\end{array}$ \\
\hline $\begin{array}{l}\text { R.A. Belei } \\
\text { M.S.Tavares } \\
\text { N.S. Paiva }\end{array}$ & $\begin{array}{c}\text { Lixo Hospitalar: qual o verdadeiro } \\
\text { perigo? }\end{array}$ & $\begin{array}{c}\text { Unopar Científica } \\
\text { Ciências Biológicas } \\
\text { e da Saúde }\end{array}$ & v.2, n.1, out/2000. \\
\hline M.H.D.M.Guariente & Destaque Nursing do HURNP & Revista Nursing & $\begin{array}{c}\text { v.3, n.21, p.s, } \\
\text { fev/2000 }\end{array}$ \\
\hline M.C.L. Haddad & $\begin{array}{l}\text { Qualidade de vida do Trabalhador de } \\
\text { Enfermagem }\end{array}$ & $\begin{array}{l}\text { Revista Espaço } \\
\text { para Saúde }\end{array}$ & $\begin{array}{l}\text { on-line, v.1, n.2, } \\
\text { p. } 75-88, \text { jun/2000. }\end{array}$ \\
\hline M.C.L. Haddad & Como eu cuido de quem cuida? & Revista Nursing & v.4, n.37, jun/2001 \\
\hline $\begin{array}{l}\text { C.C.T. Kuwabara } \\
\text { M.C.L. Haddad }\end{array}$ & $\begin{array}{c}\text { Enfermagem reduz custos e Desperdicios } \\
\text { de Materiais através de Gerenciamento } \\
\text { Implantado }\end{array}$ & Revista Nursing & $\begin{array}{l}\text { v. } 4, \text { n. } 40, \text { p.04 } \\
\text { set } / 2001\end{array}$ \\
\hline $\begin{array}{l}\text { R.L.S. Depieri } \\
\text { M.H.D.M. Guariente }\end{array}$ & $\begin{array}{c}\text { Qualidade do Serviço de Enfermagem } \\
\text { Ambulatorial: um Instrumento de } \\
\text { Avaliação }\end{array}$ & $\begin{array}{l}\text { Revista Espaço } \\
\text { para Saúde }\end{array}$ & $\begin{array}{l}\text { v.3, n.1, } \\
\text { dez/2001 }\end{array}$ \\
\hline $\begin{array}{l}\text { M.V. Fernandes } \\
\text { A.S. Batista } \\
\text { M.A.N. Leite }\end{array}$ & $\begin{array}{c}\text { Endomarketing - Uma Possibilidade nos } \\
\text { Serviços de Saúde }\end{array}$ & $\begin{array}{l}\text { Revista Espaço } \\
\text { para Saúde }\end{array}$ & $\begin{array}{l}\text { on-line, v.3, } \\
\text { n.2, jun } / 2002\end{array}$ \\
\hline $\begin{array}{l}\text { M.V. Fernandes } \\
\text { M.C.L. Haddad } \\
\text { M.H.D.M. Guariente }\end{array}$ & $\begin{array}{c}\text { Enfermeiros de Hospital de Ensino na } \\
\text { Prestação de Serviços }\end{array}$ & Revista Nursing & $\begin{array}{l}\text { v. } 6, \mathrm{n} .55 \\
\text { dez/2002 }\end{array}$ \\
\hline
\end{tabular}

Figura 6. Distribuição das Publicações realizadas por enfermeiras assistenciais do HURNP na Linha de Pesquisa Gerenciamento dos Serviços de Saúde e de Enfermagem, segundo autoria, título, veículo de divulgação e demais indicações bibliográficas, 1999-2002, Londrina-Pr, 2003. 
Os resultados apresentados nos Quadros 1 a 5 evidenciam que a produção científica publicada trata de uma variedade de temas e abrange cinco linhas de pesquisa de duas áreas, a Assistencial e a Organizacional, que compõem o esquema categorial de referência, citado na introdução do texto.

A área Assistencial, que agrega as Linhas de Pesquisa 2.1-Processo de Cuidar em Saúde e Enfermagem e a Linha 2.2-Saúde e Qualidade de vida, concentra $14(38,9 \%)$ publicações. O realce das produções escritas são em torno de temas que discutem o saberfazer profissional, relativo ao contexto dos assistidos (CARVALHO, 2000).

A área Organizacional, que engloba as Linhas de Pesquisa 3.2-Políticas e Práticas de Educação e Enfermagem, a Linha 3.3-Produção Social e Trabalho em Saúde e Enfermagem e a Linha 3.4Gerenciamento dos Serviços e de Enfermagem, detém $22(61,1 \%)$ produções. Essas linhas correspondem a estudos relacionados à realidade do cuidar/cuidados no mundo da Enfermagem (CARVALHO, 2000).

Nenhuma das produções publicadas contemplou o campo temático Profissional que se refere às principais disciplinas, conteúdos teóricos e experiências que se ajustam ou compõem a parte do saber profissional (CARVALHO, 2000).

Estudiosos vêm discutindo sobre as linhas de pesquisa em evidência nos trabalhos produzidos por enfermeiras assistenciais, reforçando os achados deste estudo.

Rodrigues e Bagnato (2003) descrevem, com base em pesquisa na qual analisaram artigos publicados nos últimos cinco anos, em três periódicos de circulação nacional., responsáveis pela divulgação de grande parte da produção de conhecimentos de Enfermagem, que existe uma certa tendência das temáticas voltadas para a assistência.

Leão (2005b) apresenta um levantamento, em que mostra que áreas de interesse na produção dos enfermeiros de hospital privado se destinaram, principalmente, à saúde do Adulto e à área da Assistência à Criança, tendo encontrado, nessas áreas, diversos temas relacionados ao cuidar/ cuidado.

Dyniewicz (2003) verificou que, nas produções de enfermeiras assistenciais de hospital público, os temas de interesse relacionam-se às linhas de pesquisa do processo de cuidar em enfermagem, práticas de educação em saúde, gerenciamento dos serviços de enfermagem e comunicação em enfermagem.

Em vista deste quadro e levando em conta o desenvolvimento da habilidade investigativa entre as enfermeiras pesquisadoras, informantes deste estudo, é importante agrupá-las por afinidade temática, para que elas possam aprofundar e avançar no processo investigativo instaurado neste contexto organizacional e, assim, pautar o cuidado prestado nos conhecimentos científicos construídos e adquiridos. A criação de grupos de pesquisa pode responder com resolutivamente a essa questão além de possibilitar uma produção efetivamente coletiva (RODRIGUES; BAGNATO, 2003).

Ainda, a fim de identificar os artigos pela classificação dos periódicos, seguimos a descrição de Dyniewicz (2003) que apresenta as categorias: Relatos de Experiência, Pesquisas, Reflexões e Revisão.

Os Relatos de Experiência são definidos como a narrativa de experiências da vida profissional, com a construção de conhecimentos provenientes do cotidiano e alicerçados em bibliografia que os confirme (DYNIEWICZ, 2003).

A autora ainda explica que neste caminho estão as Reflexões e as Revisões "como estudos preliminares sobre um assunto, circunscrevendo e analisando seus aspectos, contribuindo para uma pesquisa futura mais estruturada". Tais Revisões aproximam-se da pesquisa bibliográfica ao basearse numa vasta literatura para estudar e analisar as contribuições deste acervo em um determinado assunto, exigindo especificações sobre procedimentos utilizados para coleta do acervo 
recolhido e análise desta literatura (DYNIEWICZ, 2003, p.118-119).

Encontramos, nos artigos publicados pelas enfermeiras do HURNP, a seguinte freqüência por categoria, segundo descrição de Dyniewicz (2003): $16(44,44 \%)$ Pesquisas; 10 (27,7\%) Relatos de Experiência; $6(16,65)$ Revisões e $4(11,1 \%)$ Reflexões.

Estes dados confirmam a afirmação de que práticas educativas em Metodologia da Pesquisa, por meio de atividades individuais e em grupo, e voltadas para o desenvolvimento de competências em investigação científica, despertaram o interesse das enfermeiras na elaboração e divulgação de relatos de experiências e pesquisas descritivas e exploratórias (DYNIEWICZ, 2005; FONSECA, 2005; LEÃO, 2005a).

A Figura 7 apresenta a Produção Científica das enfermeiras assistenciais do HURNP, premiada em eventos.

\begin{tabular}{|c|c|c|c|c|}
\hline AUTORES & TíTULOS & EVENTO/LOCAL & ANO & CATEGORIA \\
\hline $\begin{array}{l}\text { M.C.L. Haddad } \\
\text { C. Kuwabara }\end{array}$ & $\begin{array}{l}\text { Inserção do enfermeiro } \\
\text { no controle de materiais } \\
\text { e equipamentos de um } \\
\text { hospital-escola-pública }\end{array}$ & $\begin{array}{c}7^{\circ} \text { ENFTEC } \\
\text { S. Paulo }\end{array}$ & 2000 & $\begin{array}{c}\text { Melhor Tema } \\
\text { Livre }\end{array}$ \\
\hline $\begin{array}{l}\text { M. Fernandes } \\
\text { M.C.L. Haddad } \\
\text { M.H.D.M. Guariente }\end{array}$ & $\begin{array}{c}\text { Enfermeiros de Hospital } \\
\text { de ensino na Prestação } \\
\text { de Serviço }\end{array}$ & $\begin{array}{c}\text { VI Encontro de } \\
\text { Enfermeiros em hospitais } \\
\text { de Ensino do Estado de } \\
\text { São Paulo. São Paulo }\end{array}$ & 2002 & $\begin{array}{c}1^{\circ} \text { Lugar } \\
\text { Modalidade } \\
\text { Pôster }\end{array}$ \\
\hline $\begin{array}{l}\text { M.M.B. Oliveiraa } \\
\text { M.T.O.Vannuchi } \\
\text { J.O.P. Popper } \\
\text { J.Q. Borges } \\
\text { M.P. Silva } \\
\text { L.Y. Nishikawa }\end{array}$ & $\begin{array}{c}\text { Produção de leite Humano } \\
\text { Ordenhado "Zero Defeito": } \\
\text { uma proposta }\end{array}$ & $\begin{array}{l}11^{\circ} \text { Encontro Paulista } \\
\text { Aleitamento Materno } \\
4^{\circ} \text { Encontro Paulista } \\
\text { de Banco de Leite } \\
\text { Humano. São Paulo }\end{array}$ & 2000 & $\begin{array}{c}\text { Menção } \\
\text { Honrosa } \\
2^{\circ} \text { Colocação }\end{array}$ \\
\hline $\begin{array}{l}\text { E.H.T. Anami } \\
\text { A.R. Oliveira } \\
\text { A.G. Elias } \\
\text { C. Gpion } \\
\text { L.Q. Cardoso }\end{array}$ & $\begin{array}{c}\text { Reposição Volêmica } \\
\text { utilizando peso diário } \\
\text { e monitorização } \\
\text { hemodinâmica - relato } \\
\text { de caso }\end{array}$ & $\begin{array}{l}42^{\circ} \text { Congresso da } \\
\text { Associação Médica de } \\
\text { Londrina. Londrina-Pr }\end{array}$ & 2000 & $\begin{array}{l}2^{\circ} \text { Lugar Pôster } \\
\text { da Área Clínica }\end{array}$ \\
\hline $\begin{array}{l}\text { L.T. Tatakiharo } \\
\text { M.A.B. Nascimento } \\
\text { R.L. Depieri }\end{array}$ & $\begin{array}{c}\text { Atuação da } \\
\text { Enfermagem } \\
\text { na Radiologia }\end{array}$ & $\begin{array}{c}\text { VII Jornada Paranaense } \\
\text { de Radiologia } \\
\text { Foz do Iguaçu - Pr }\end{array}$ & 1999 & $\begin{array}{l}\text { Menção } \\
\text { Honrosa }\end{array}$ \\
\hline $\begin{array}{l}\text { R.A.Bclei } \\
\text { C. Grion }\end{array}$ & $\begin{array}{l}\text { Mortalidade e aumento } \\
\text { do tempo de internação em } \\
\text { pacientes portadores de } \\
\text { bactérias multiresistentes }\end{array}$ & $\begin{array}{l}42^{\circ} \text { Congresso da } \\
\text { Associação Médica de } \\
\text { Londrina. } \\
\text { Londrina - Pr }\end{array}$ & 2000 & $\begin{array}{c}1^{\circ} \text { Lugar Pôster } \\
\text { Área Clínica }\end{array}$ \\
\hline
\end{tabular}

Figura 7. Distribuição da Produção Científica das enfermeiras assistenciais do HURNP, premiada em eventos, segundo Autoria, Título, Evento Científico, Ano e Categoria do Prêmio referente ao quadriênio 1999 - 2002, Londrina-Pr, 2003. 
Ainda com respeito à produção científica premiada no quadriênio, observamos, conforme descrições da Figura 7, que os trabalhos abrangem diferentes temáticas, em virtude da inclusão das enfermeiras em novas áreas de atuação, como a área de controle de materiais e prestação de serviço, como também da assistência em saúde e enfermagem em áreas especializadas, tais como Comissão de Controle de Infecção Hospitalar, Aleitamento Materno, Unidade de Terapia Intensiva e Unidade de Radiologia.

\section{Considerações Finais}

A análise empreendida possibilitou lançar um olhar reflexivo sobre a produção referente às atividades investigativas das enfermeiras assistenciais apoiadas por um núcleo de pesquisa.

Observamos que as enfermeiras do HURNP, nos anos de 1999 a 2002, isoladamente ou em parceria com os professores e alunos de Enfermagem publicaram 36 artigos.

Verificamos também que os artigos publicados por essas enfermeiras, em diferentes veículos de divulgação, indexados ou não, tratam de assuntos relacionados às Linhas de Pesquisa do campo temático Assistencial e Organizacional.

Embora esse quadro seja animador, há ainda um longo caminho pela frente para uma melhora efetiva na prática da investigação científica por enfermeiras assistenciais que evidenciam diferentes aspectos, desde a qualidade e rigor metodológico até a percepção da necessária construção e utilização de novos conhecimentos na própria atuação profissional.

Vivenciamos momento profícuo e oportuno para a efetivação de propostas inovadoras que dinamizem o processo de educação permanente nas instituições de saúde. Com isso, será dado estimular e promover o desenvolvimento da competência em pesquisa, a fim de atender as necessidades da prática assistencial.
Nesse sentido, o incentivo e a mediação da assessoria ou de um setor equivalente contribuiram para um salto qualitativo necessário da produção, divulgação e aplicação de conhecimentos na área.

\section{Referências}

ADAMI, N. P.; SALZANO, S. D.T.; CASTRO, R. A. P.; STEFANELLI, M. C. Situação da pesquisa em enfermagem em hospitais do município de São Paulo. Revista Latino-americana de Enfermagem, Ribeirão Preto, v.4, n.1, p.5-20, jan./fev. 1996.

AGUDELO, M. C. C. Trends and priorities in nursing research. Revista Latino-americana de Enfermagem, Ribeirão Preto, v.12, n.4, p.583588, jul./ago. 2004.

BRASIL. Ministério da Saúde. Secretaria de Ciência e Tecnologia. Agenda Nacional de Prioridades de Pesquisa. Brasília, DF, 2004.

CALIRI, M. H. L. A utilização da pesquisa na prática clínica de enfermagem: limites e possibilidades. 2002. Tese. (Livre Docência) Escola de Enfermagem de Ribeirão Preto, Universidade de São Paulo, Ribeirão Preto.

CARVALHO, V. Linhas de Pesquisa e Prioridades de Enfermagem-Proposta com distinção gnoseológica para o agrupamento da produção científica da pós-graduação em Enfermagem. Mimeo. In: ENCONTRO DE PESQUISA DA RENE, 2., 2000, Salvador. Anais... Salvador, 2000.

CASSIANI, S. H. B.; PASSARELLI, L. R. Pesquisa em enfermagem: um processo de ação do enfermeiro. Revista Gaúcha de Enfermagem, Porto Alegre, v.20, n.1, p.91-110, jan. 1999.

CASTILHO, V.; KURCGANT, P. O vivencial de enfermeiros: assistenciais no desenvolvimento de pesquisa nas organizações hospitalares. $O$ Mundo 
da Saúde, São Paulo, v.23, n.3, p.159-166, maio/ jun. 1999.

CIANCIARULLO, T. I. A questão da saúde, da pesquisa e do poder - um problema para os enfermeiros. Revista da Escola de Enfermagem da USP, São Paulo, v.26, n.esp., p.153-156, out. 1992.

COSTA, R. S.; CARVALHO, D. V. Análise da produção científica dos enfermeiros de Minas Gerais publicada em periódicos de enfermagem. Revista Latino-americana de Enfermagem, Ribeirão Preto, v.9, n.5, p.19-25, set./out. 2001.

DYNIEWICZ, A. M. Metodologia da pesquisa para enfermeiros: práticas educativas em hospital universitário. 2003. Tese (Doutorado)- Escola Paulista de Medicina, Universidade Federal de São Paulo, 2003.

DYNIEWICZ, A. M.; GUTIÉRREZ, M. G. R. Metodologia da pesquisa para enfermeiras de um hospital universitário. Revista Latino-americana de Enfermagem, Ribeirão Preto, v.13, n.3, p.354-363, maio/jun. 2005.

FONSECA, A. S. Assessoria em Pesquisa: a produção científica e a busca da qualidade. In: SEMINÁRIO NACIONAL DE PESQUISA EM ENFERMAGEM, 13., 2005, São Luiz. Anais ... São Luiz: ABEn, 2005.

CD-ROM.

GUTIERREZ, M. G. R.; LEITE, J. L.; PAGLIUCA, L. M. F; ERDMANN, A. L. Os múltiplos problemas pesquisados e a pesquisar na enfermagem. In: SEMINÁRIO NACIONAL DE PESQUISA EM ENFERMAGEM, 11., 2001, Belém. Anais... Belém: ABEn, 2001.

HADDAD, M. C. L.; GUARIENTE, M. H. D. M. A inserção do método científico na prática e na reflexão dos enfermeiros de campo. Revista Nursing, São Paulo, v.3, n.27, p.16-18, ago. 2000.
LEÃO, E. R. Assessoria de pesquisa científica: a experiência de um triênio (2002-2004). In: SEMINÁRIO NACIONAL DE PESQUISA EM ENFERMAGEM, 13., 2005, São Luiz. Anais ... São Luiz: ABEn, 2005a.

CD-ROM.

LEÃO, E. R. Assessoria de pesquisa científica: produção científica dos enfermeiros de uma instituição privada. Cadernos - Centro Universitário São Camilo, São Paulo, v.11, n.2, p.82-87, abr./jun. 2005b.

LOPES, C. M. Aplicação de resultados de pesquisa na prática da enfermagem. São Paulo: Sarvier, 1993.

MANCIA, J. R.; RAMOS, F. R. S. Pontos críticos na produção científica de enfermagem - os trabalhos submetidos à REBEn. Revista Brasileira de Enfermagem, Brasília, v.55, n.2, p.163-168, mar./ abr. 2002.

MARZIALE, M. H. P. Diretivas para o desenvolvimento de pesquisas em saúde no Brasil. Não publicado. Anotações de relatos orais dos participantes. Revista Latino-americana de Enfermagem, Ribeirão Preto, v.12, n.4, p.577-578, jul./ago. 2004.

Mendes (1991) completar referência citada no texto

RODRIGUES, R. M.; BAGNATO, M. H. S.

Pesquisa em enfermagem no Brasil:

problematizando a produção de conhecimentos.

Revista Brasileira de Enfermagem, Brasília, v.56,

n.6, p.646-650, nov./dez. 2003.

ROSAS, P. Instruções redatoriais e a indexação em publicação periódica. In: GOLDEMBERG, S.; GUIMARÃES, C. A.; CASTRO, A. A. (Org.). 
Elaboração e apresentação de comunicação científica. São Paulo: Metodologia, 2001. Disponível em: <http://www.metodologia.org>. Acesso em: 15 out. 2005.

SALZANO, S. D. T.; STEFANELLI, M. C.; ADAMI, N. P.; JORGE, A. L. S.; CASTRO, R. A. P.; SILVA, I. A. Produção científica publicada pelos enfermeiros assistenciais de hospitais públicos e privados do município de São Paulo. Revista da Escola de Enfermagem da USP, São Paulo, v.32, n.1, p.2-8, jan. 1998.

SECAF, V. Artigo científico: do desafio à conquista. São Paulo: Reis Editorial, 2000.

TASCA, A.; KOHLRAUSCH, E.; PASKULIN, L.; GALPERIM, M.; MOSCHINI, M.; PEREIRA, R.; BERCINI, R.; SCAIN, S. Vivenciando a integração docente-assistencial através da pesquisa. Revista Gaúcha de Enfermagem, Porto Alegre, v.17, n.1, p.66-69, jan. 1996. 